\title{
Entrevista clínica y valoración funcional del suelo pélvico
}

\author{
Clínical interview and functional assessment of pelvic floor
}

\author{
S. Martínez Bustelo, A. Ferri Morales, S. Patiño Núñez, S. Viñas Diz, A. Martínez \\ Rodríguez
}

\begin{abstract}
Resumen
Los programas de reeducación fisioterápica de la región perineal deben basarse en una completa entrevista clínica y valoración funcional de la esfera abdomino-pelvi-perineal. A partir de los datos obtenidos, estableceremos los objetivos de tratamiento y la selección de las técnicas manuales e instrumentales para alcanzar los objetivos planteados. Además de la anamnesis, realizaremos una exploración física exhaustiva del suelo pélvico, que comprenderá la exploración visual y palpatoria, la valoración muscular, la valoración de la estática de los órganos pélvicos y la exploración neurológica; completaremos la valoración específica del suelo pélvico con la valoración abdominal, del diafragma y de la región lumbopélvica.
\end{abstract}

\begin{abstract}
All the physical therapist reeducation programs of the pelvic floor must be based on a thorough clinical interview and functional assessment of the abdominal region, the pelvis and the pelvic floor. From all these data, we will establish the goals o/ the treatment and we will select the manual and instrumental technics to reach the aims we have set out. Besides the clinical interview, we must carry out a comprehensive physical examination of the pelvic floor, which covers the visual and palpable examination, the muscle assessment, the assessment of the biomechanical position of the pelvic organs, and the neurological examination; we must complete the specific assessment of the pelvic floor with the examination of the abdominal region, the diaphragm and the low back
\end{abstract}

Palabras clave

Fisioterapia; Valoración; Suelo pélvico.

Keywords

Physical Therapy; Assessment; Pelvic floor.

\section{Introducción}

Tanto las técnicas manuales como instrumentales empleadas en la reeducación funcional del suelo pélvico, han demostrado ser eficaces y eficientes en diversos estudios científicos ${ }^{1}$. Sin embargo, para alcanzar estos resultados la entrevista clínica y la valoración funcional debe constituir el primer paso de toda reeducación perineo-esfinteriana, puesto que a partir de los datos obtenidos, se establecerán los objetivos del tratamiento fisioterápico en función de los cuales seleccionaremos las técnicas y parámetros más apropiados en cada caso clínico.

La entrevista clínica y valoración del Suelo Pélvico, comprende una serie de procedimientos cualitativos y cuantitativos que valoran desde el punto de vista funcional los parámetros biomecánicos abdomino-pelvi-perineales.

El protocolo de valoración fisioterápica del suelo pélvico comprenderá en primer lugar una anamnesis exhaustiva, seguida de una exploración física detallada de la región perineal y de la estática lumboabdormno-pélvica, que nos permitirán valorar: la existencia de incontinencia urinaria, el tipo de incontinencia y la causa de la misma, y la presencia de otras disfunciones asociadas, tales como prolapsos de los órganos pélvicos, disfunciones sexuales, o disuria (dolor durante la micción) ... 


\section{Anamnesis}

La anamnesis constituye el primer paso de la valoración del suelo pélvico. Consistirá en un conjunto de cuestiones acerca de los datos administrativos del paciente, de cuál es el motivo de la consulta, los antecedentes médicos y obstétricos de la paciente, su profesión y actividades deportivas que realiza, así como de las características de los episodios de incontinencia que padece. Todas estas cuestiones deben de recogerse en un formulario protocolizado que además de facilitamos la valoración, resulta muy práctico para el análisis estadístico posterior de los casos clínicos.

En la mayor parte de las ocasiones, una vez finalizada la anamnesis ya disponemos de suficientes datos para identificar si el paciente sufre de una incontinencia urinaria de esfuerzo, mixta o de urgencia.

\section{Motivo de la consulta}

El motivo más frecuente por el que una mujer suele consultar a un fisioterapeuta dentro del campo de la reeducación perineo-esfinteriana, es sin duda la incontinencia urinaria, siendo el período postparto y menopáusico los de mayor incidencia. Estos datos se demuestran en la encuesta realizada por Wilson sobre 1505 mujeres tres meses después del parto con 34,3\% de incontinentes ${ }^{2}$, mientras que el 47,6\% de mujeres tras la menopausia refieren escapes involuntarios de orina según Minaire ${ }^{3}$.

En otras ocasiones el motivo de consulta es un prolapso pélvico, presente en el $30 \%$ de las mujeres entre 29 y 59 años según un estudio sueco realizado sobre 487 personas por Samuelsson y Victor ${ }^{4}$.

Sin embargo, este tipo de disfunciones se asocian frecuentemente a dolores perineal es, incontinencias fecales, o disfunciones sexuales, que la paciente no suele referir inicialmente y que en cambio pueden suponer en ocasiones un mayor problema social y personal que la propia incontinencia urinaria.

\section{Antecedentes médicos y obstétricos}

Dentro de los antecedentes obstétricos, hemos de preguntar a la paciente acerca de diferentes aspectos que su- ponen un mayor riesgo perineal, tales como:

- Número de embarazos y partos.

- Tipo de parto, vaginal o por cesárea.

- El uso de fórceps o ventosa.

- La duración de las fases de dilatación y expulsivo.

- Desgarros o episiotomías perineales.

- Episodios de incontinencia transitoria tras el parto.

En el postparto inmediato se estima que el $50 \%$ de las mujeres con parto vaginal presentan una incontinencia urinaria de esfuerzo transitoria, que evoluciona hacia la regresión o curación espontánea en un 60-80\% de los casos tras los dos meses del parto. Sin embargo, estas incontinencias transitorias son reflejo de una alteración perineal que representa un riesgo aumentado en un 2,5 de desarrollar incontinencia ulterior ${ }^{5}$.

Sin embargo, no solo las mujeres con partos vaginales presentan riesgo de padecer incontinencia urinaria postparto. Según un estudio realizado en 15.307 mujeres, se observó que en comparación con las mujeres nulíparas, las que habían dado a luz mediante cesárea tenían una probabilidad de 1,5 de experimentar cualquier tipo de incontinencia. La explicación radica en que no solo el parto, sino también el peso del feto y la alteración postural durante la gestación son factores de riesgo de padecer una incontinencia de orina en el periodo postparto al sobrecargar el periné anterior ${ }^{6}$. A la vista de los resultados, queda patente la importancia de conocer los antecedentes obstétricos de la paciente durante la fase de la anamnesis.

Respecto a los antecedentes médicos, se anotarán todos aquellos datos relativos a:

- Patologías y/o cirugías ginecológicas.

- Patologías neurológicas y/o con denervación muscular.

- Patologías respiratorias que provoquen una hiperpresión intraabdominal de forma crónica, como puede ser la bronquitis crónica o las alergias.

- Patologías digestivas que cursen con estreñimiento crónico.

- Patologías psicológicas. 
Otro dato de gran relevancia es el tipo de Medicamentos que la paciente toma frecuentemente; hemos de tener en cuenta que numerosos medicamentos actúan sobre el sistema urinario inferior, como los diuréticos, los hipotensores, los vasodilatadores, los neurolépticos, los miorrelajantes, y los antidepresivos, pudiendo dificultar el éxito del tratamiento fisioterápico.

En un estudio realizado por Minaire, señaló que el $43 \%$ de sus pacientes remitidos a fisioterapia para la reeducación del periné tomaban uno o varios medicamentos de los anteriormente señalados ${ }^{7}$.

\section{Profesión y actividades deportivas}

Dentro de la anamnesis se preguntará acerca del tipo de actividad profesional que realiza la paciente, dado que todas aquellas profesiones en donde se permanece muchas horas de pie, y especialmente las que requieren el transporte de cargas son las que suponen un mayor factor de riesgo perineal. Entre las profesiones más lesivas para el suelo pélvico se encuentran: las dependientas, las profesoras, las peluqueras, las auxiliares de clínicas y celadoras, las cuidadoras de ancianos y niños, las cantantes, las bailarinas y profesoras de aeróbic, etc.

Otro factor muy importante es La actividad deportiva que desarrolla la paciente, la frecuencia y la intensidad de entrenamiento. En general todos aquellos deportes que provoquen un aumento importante de la presión intraabdominal, suponen un factor de riesgo perineal. Entre los deportes más hiperpresivos destacan: los ejercicios abdominales clásicos, el atletismo, el baloncesto, el aeróbic, el tenis, con valores de presión intraabdominal superiores a $110 \mathrm{~mm} \mathrm{de} \mathrm{Hg}^{8}$, mientras que la natación y el ciclismo son los que menos presión intrabdominal generan.

Según un estudio realizado por Elleuch M et al sobre 105 alumnas de Educación física, con una media de edad de 21,5 años que practicaban deporte de competición, el 62,8\% presentaban incontinencia de orina de esfuerzo relacionada con las actividades deportivas ${ }^{9}$.

\section{Características de los episodios de incontinencia}

Una vez analizados los antecedentes personales y el estilo de vida de la paciente, se inicia la valoración específica de los episodios de incontinencia, que nos permitirán identificar no solo el tipo de fuga de orina, sino también los signos asociados y la cuantificación de dicha pérdida.

Para conocer el tipo de la fuga de orina, preguntaremos a la paciente sobre cuándo se producen las pérdidas, con qué frecuencia, y los factores que desencadenan o se asocian a las fugas.

En el caso de una incontinencia de esfuerzo, las fugas se producen a lo largo del día sincronizadas con determinados esfuerzos que provocan una aumento de la presión abdominal, como la tos, el estornudo, el levantar un peso, correr, etc. e incluso la marcha o el simple cambio de posición. Algunas pacientes refieren que la frecuencia de los escapes aumenta en días previos a la menstruación y durante la misma, lo que puede tener su explicación en la disminución del nivel de estrógenos que tiene consecuencias en la presión de cierre uretral al disminuir la vascularización de la capa submucosa ${ }^{10}$,

Sin embargo, las pacientes con incontinencias de urgencia, refieren episodios de incontinencia tras una necesidad repentina e imperiosa de orinar, tanto durante el día como durante la noche. Esta necesidad urgente aparece frecuentemente asociada a factores psicológicos corno las épocas de gran stress, episodios de risas a carca- jadas, etapas de depresión, asociado al frío, o incluso a acciones como abrir la puerta de casa (Síndrome del picaporte), estar cerca del cuarto de baño, escuchar o tocar el chorro del agua del grifo abierto. También en este tipo de incontinencia las pacientes refieren que los síntomas se agravan durante los días de menstruación (tabla 1). 
Tabla 1. Características más relevantes de cada tipo de incontinencia urinaria

\begin{tabular}{lcc}
\hline Síntomas & I. U. esfuerzo & I U. urgencia \\
\hline & & \\
Fugas con esfuerzos & +++ & + \\
Sincronizadas con esfuerzos & +++ & 0 \\
Diferidas factor causal & 0 & 0 \\
Cambios de posición & +++ & + \\
Durante la marcha & ++ & + \\
Necesidades urgentes & + & +++ \\
Fugas nocturnas & 0 & +++ \\
Eretismo al agua, frío & + & ++ \\
Período menstrual & ++ & +++ \\
\end{tabular}

En cuanto a la frecuencia de micción, debemos de valorar no solo el número de micciones diarias, sino además el aporte hídrico de la paciente, y el tipo de bebida, ya que los líquidos con cafeína como el café, té, coca cola, y las bebidas alcohólicas, tiene un efecto excitador sobre la vejiga urinaria. Para el estudio y seguimiento de la frecuencia urinaria, resulta de gran utilidad el manejo de los calendarios o diarios miccionales, en donde la paciente recoge diariamente la cantidad y el tipo de líquidos ingeridos, además del número de micciones voluntarias, el volumen de orina evacuado, los episodios de escape o fuga urinaria y el motivo que los produjo (tos, estornudo, urgencia, etc) y la cantidad y tipo de protectores absorbentes usados a lo largo del día (fig. 1).

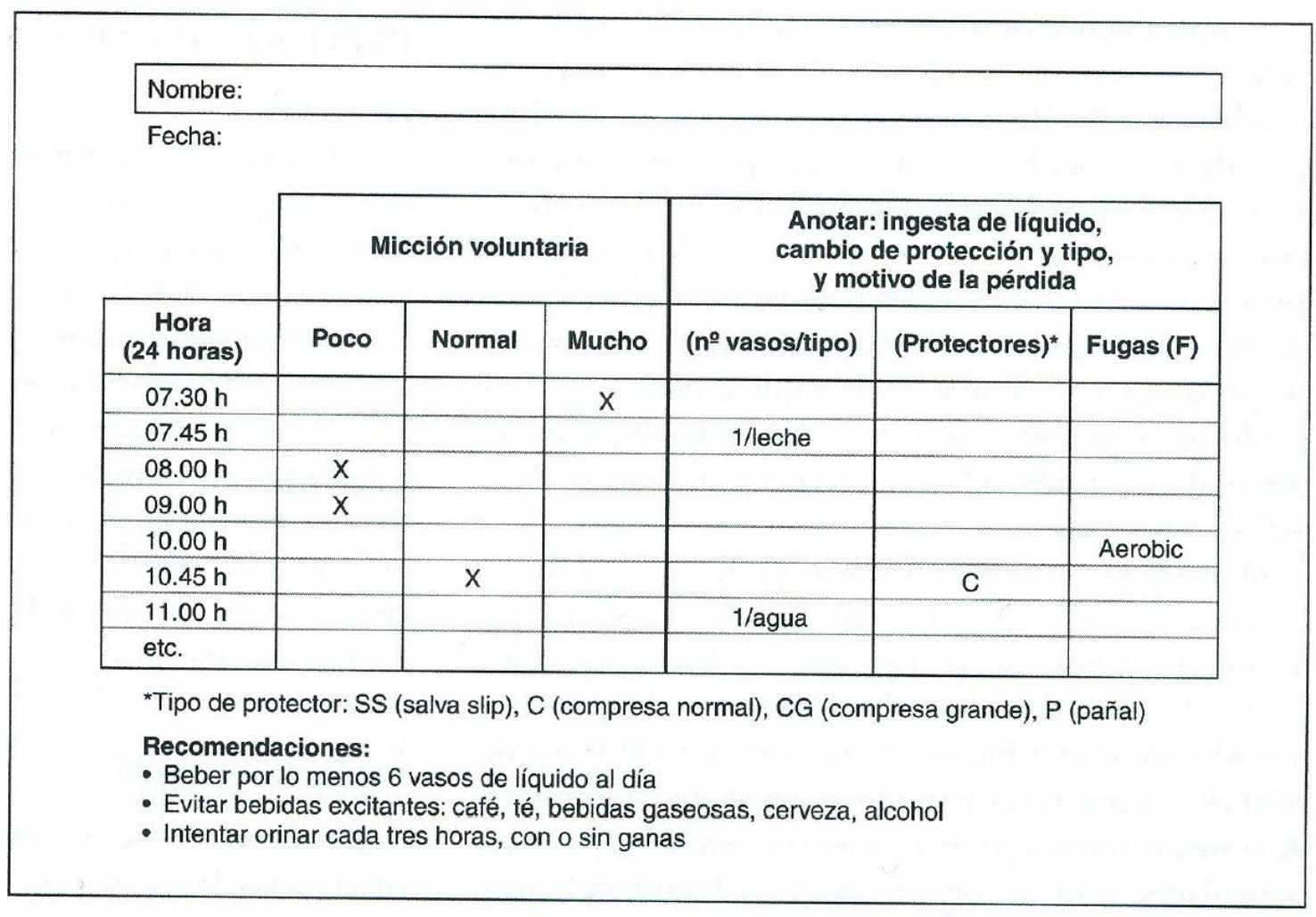

Fig. 1. Ejemplo de diario miccional 
En condiciones normales, el número de micciones nocturnas debe ser cero, o al menos no levantarse de forma asidua por la noche para ir a orinar. Durante el día normalmente el tiempo entre una micción y la siguiente debe ser de unas tres horas, lo cual implica entre 5 y 6 micciones diarias. Sin embargo, las pacientes con incontinencia de urgencia refieren polaquiuria tanto diurna como nocturna, siendo de gran interés conocer el tiempo de seguridad del que dispone la paciente entre la primera sensación de urgencia y el episodio de incontinencia. En el caso de la incontinencia de esfuerzo, en muchas pacientes podemos observar una polaquiuria que podemos denominar de "precaución", dado que por miedo a la fuga vacía su vejiga frecuentemente.

Dentro de los signos asociados, en ocasiones la mujer presenta interrupciones durante la micción, orinando en varias veces en vez de una sola; este tipo de sintomatología refleja una posible disinergia entre el músculo detrusor de la vejiga y el esfínter liso uretral. En los casos de prolapsos de los órganos pélvicos, la paciente precisa a veces de una ayuda manual que reduzca el prolapso y le permita orinar. Esto es debido a la resistencia que pue- de generar un prolapso pélvico instaurado al paso de la orina.

También debemos interesarnos sobre si existen escozores o picores durante la micción, es decir disuria; este síntoma se debe frecuentemente a una cistitis o infección de orina, por lo que debemos de posponer todo tratamiento fisioterápico hasta la resolución de la infección, y derivar la paciente hacia el médico para que realice un cultivo y prescriba el tratamiento correspondiente. Así mismo. es frecuente la disuria en casos graves de prolapsos, con la característica que se agrava a lo largo del día o tras permanecer mucho tiempo de pie por el efecto del peso sobre el suelo pélvico y los órganos de la pelvis.

Al objeto de cuantificar las pérdida de orina, la Sociedad Internacional para la Continencia propone la utilización del denominado "Pad Test" o "Test de la Compresa". Este test se realiza a lo largo de una hora, con la vejiga llena y tras ingerir el paciente un volumen de $500 \mathrm{cl}$ de agua en un período máximo de 15 minutos. A la media hora el paciente debe realizar las siguientes actividades: subir un piso de escaleras, levantarse y sentarse diez veces consecutivas, toser con fuerza diez veces, correr sobre el mismo punto durante un minuto, agacharse a coger un objeto del suelo cinco veces consecutivas, y finalmente lavarse las manos en agua fría corriente durante un minuto.

Terminado el test, se debe pesar la compresa en una báscula digital antes y después de los ejercicios para cuantificar la fuga de orina. Diferencias de peso de $+1 \mathrm{o}+2 \mathrm{~g}$ se consideran normales puesto que pue- den incluso deberse a la propia sudoración del periné o al flujo vaginal. Los resultados del test se expresarán en gramos de orina perdidos durante una hora. En un estudio realizado por Sutherst et al en una población de 100 mujeres incontinentes y 50 mujeres continentes, la pérdida media de orina siguiendo el Test de la compresa es de $0,8 \mathrm{~g}$ en las pacientes con control normal de la orina, de $25,7 \mathrm{~g}$ en las mujeres con incontinencia urinaria de stress y de $20,7 \mathrm{~g}$ en las pacientes con incontinencia de urgencia; además observaron que mientras las pacientes con incontinencia de stress sufrían una media de 5-6 escapes a lo largo de la hora de duración del test, las mujeres con urgencia miccional solo referían 1-2 pérdidas de orina ${ }^{11}$.

\section{Exploración física}

Una vez finalizada la anamnesis se procede a la exploración física de la paciente. Dentro de la exploración física se engloba la exploración visual y palpatoria, la valoración muscular, la valoración de la estática pelvi-perineal y de la movilidad de los órganos pélvicos, la exploración neurológica, y finalmente la exploración de la estática lumbo-abdomino-pélvica.

Para la exploración física, la paciente se situará a ser posible en una camilla ginecológica en posición de litotomía, en su defecto se situará en una camilla normal en decúbito supino y con los miembros inferiores en apoyo con flexión de rodilla, ligera abducción y rotación externa de cadera.

\section{Exploración visual}

Inicialmente observaremos el estado de la piel, la presencia o no de escoriaciones, eritema, micosis, vesículas o leucorrea que nos puedan indicar una infección, en cuyo caso se suspenderá el tratamiento inmediatamente y se derivará al ginecólogo.

Es fundamental observar si existen cicatrices derivadas de una episiotomía o desgarro perineal, las cuales pueden ser las responsables de asimetrías en la musculatura perineal y de dispareunia o dolor durante el coito.

A continuación observamos la coloración que nos da una idea de la troficidad vulvovaginal, signo principal del nivel de estrógenos. En condiciones normales, la mucosa vaginal es de color rosáceo, bastante húmeda, y la vulva y labios menores muy elásticos, recuperando su posición inicial al soltar después de separados o estirarlos. En el caso que detectemos una coloración muy pálida de la mucosa, 
con un color nacarado y una pérdida de elasticidad en los labios menores es conveniente derivar la paciente al ginecólogo para que valore la necesidad de un tratamiento estrogénico, ya que el nivel de hormonas puede influir de forma considerable en la capacidad de contracción de la musculatura perineal y en el éxito del tratamiento fisioterápico.

Dos indicadores del estado de la musculatura perineal son el diámetro de apertura de la vagina y la distancia ano-vulvar. En condiciones normales, el introito vaginal se encuentra cerrado en el estado de reposo, a excepción de las mujeres multíparas que presentan una ligera apertura. Esta apertura puede clasificarse en tres grados, en función del diámetro del introito (tabla 2). Se considera una apertura normal cuando el diámetro del introito vaginal no supera los $25 \mathrm{~mm}$. La distancia ano-vulvar mide la longitud del centro tendinoso del periné, punto de inserción de la musculatura perineal, cuya longitud normal se sitúa entre los 2,5 y los $3,5 \mathrm{~cm}$ (fig. 2).

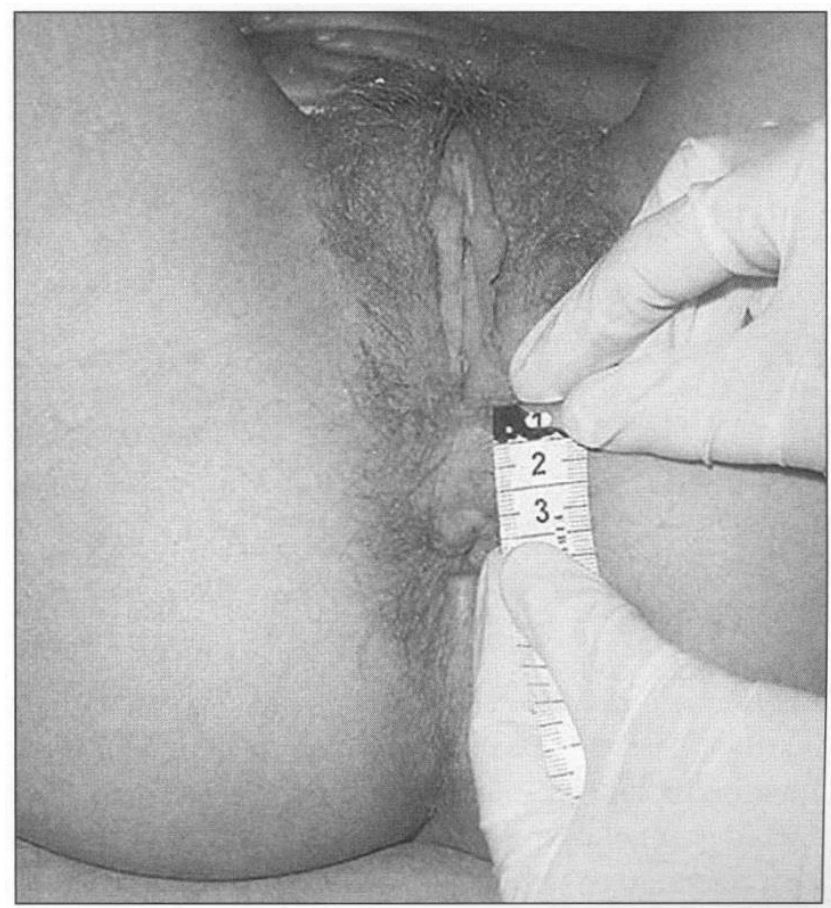

Fig. 2. Medida de la distancia ano-vulvar. Representa el espesor del núcleo fibroso central del periné. Se toma la medida desde la horquilla vulvar hasta el inicio del margen anal. Por debajo de $2 \mathrm{~cm}$ se considera patológico.

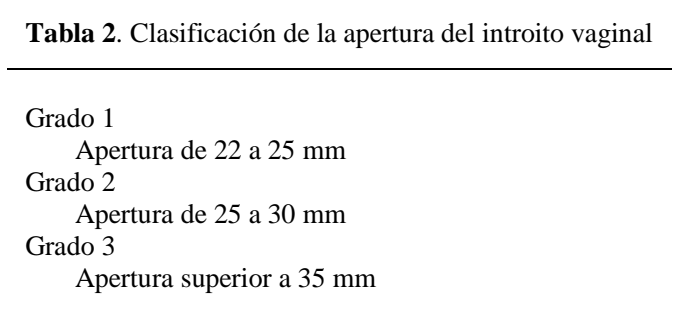




\section{Palpación}

La exploración palpatoria aporta al fisioterapeuta una información de gran relevancia acerca del estado de la musculatura y articulaciones de la pelvis, así como de la estática de los órganos de la pelvis. Mediante la palpación valoraremos el tono de la musculatura perineal, las paredes posterior y anterior de la vagina, la uretra, y el grado de movilidad del cóccix.

Para palpar el tono del periné, se introducen uno o dos dedos en la vagina con las articulaciones interfalángicas flexionadas. Mediante un movimiento dirigido hacia abajo y hacia fuera, valoraremos el grado de resistencia pasiva que ofrece la musculatura perineal al estiramiento, es decir el tono de la musculatura (fig. 3).

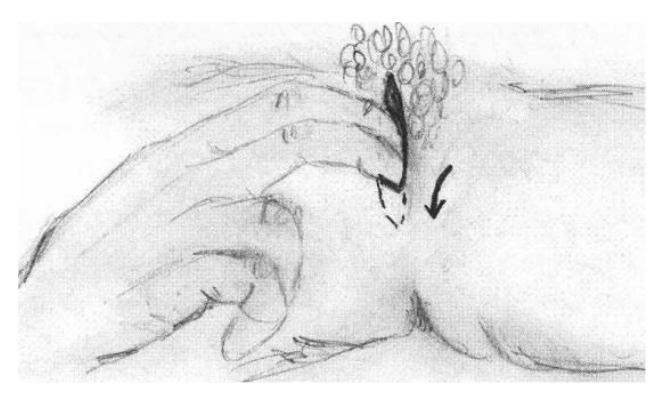

Fig. 3. Palpación del tono perineal

En condiciones normales detectaremos una resistencia de tipo "elástica", mientras que en el caso de un periné hipotónico la depresión del centro tendinoso del periné no ofrece ningún tipo de resistencia. Menos frecuente es detectar un periné hipertónico, en cuyo caso ofrecerá una gran rigidez y resistencia al estiramiento manual.

A nivel de la pared anterior vaginal palparemos la uretra, que en condiciones normales es indolora y flexible; en el caso de dolor a la palpación, puede ser un signo de uretritis. Durante la palpación de la pared anterior de la vagina a lo largo de la uretra, percibiremos una serie de estriaciones, mientras que a nivel de la vejiga, cuando ésta se encuentra llena, la pared vaginal presenta un aspecto liso, siendo capaces de detectar un surco entre la vejiga y la uretra que se corresponde con la unión uretrovesical. Durante la exploración de la pared posterior de la vagina, valoraremos la presencia de cicatrices fibróricas derivadas de una episiotornía o de desgarros perineales, responsables de posibles dispareunias y asimetrías de la musculatura.

Para la valoración de la movilidad del cóccix podemos realizado en la posición de cuadrupedia o en decúbito supino mediante una palpación anal.

\section{Valoración muscular}

Para realizar el tacto vaginal y poder valorar manualmente la musculatura perineal, introducimos el dedo medio en el introito vaginal previamente impregnado de lubricante, siguiendo siempre la dirección de la vagina oblicua hacia abajo y hacia atrás; seguidamente ejercemos una depresión caudal de la musculatura del suelo pélvico que nos permitirá introducir finalmente el dedo índice. A continuación realizamos una pronación máxima del antebrazo de manera que los dedos Índice y medio se sitúen con las articulaciones interfalángicas semiflexionadas sobre los fascículos de los músculos elevadores del ano. La valoración debe realizarse tanto de forma global como de forma individual en cada uno de los fascículos derecho e izquierdo, ya que en el caso de existir asimetrías el programa de tratamiento se centrará en mayor medida en el lado más afecto.

Solicitaremos a la paciente que intente contraer la musculatura del suelo pélvico en un intento de cerrar la vagina como si quisiera retener la orina. En otras ocasiones es preciso recurrir al símil de retener un gas para que la paciente sea consciente de qué musculatura le estamos solicitando.

Para la selección de la escala de valoración de la fuerza muscular para el suelo pélvico, nos hemos basado en un estudio realizado por Jeyaseelan, Haslam et al, en donde se recoge solo aquellas escalas cuya fiabilidad entre diferentes evaluado res haya sido demostrada científicamenre ${ }^{12}$. Dichas escalas son las desarrolladas por Worth et al en 1986 (tabla 3), Brink et al en 1989 (tabla 4), y la escala de Laycock de 1992 (tabla 5). En la tabla 3 podemos observar como la Escala de Worth es relativamente simple, 
empleando tres variables (presión o fuerza de contracción muscular, duración de la contracción y tex- tura del tejido bajo nuestros dedos) con tres grados diferentes; la presión tiene tres niveles, ligera, moderada o fuerte, pero no se proporcionan otros detalles sobre lo que el examinador debe percibir. Esta escala se realiza de forma digital con un solo dedo, normalmente el índice. La Escala de Brink se diferencia de la Escala de Worth en que, además de realizarse de forma bidigital, es más exacta en cuanto que presenta más grados y añade un nuevo grado y una nueva variable a valorar, el desplaza- miento de la musculatura en el plano vertical. Por el contrario, la Escala de Laycock describe de forma más detallada lo que el examinador debe percibir en sus dedos en cada uno de los 5 grados. Además permite grados intermedios con el empleo de los signos + y - en cada uno de los grados. Esta escala es la más empleada en el Reino Unido, si bien solo refleja el grado de fuerza de la musculatura del suelo pélvico.

Tabla 3. Escala de valoración de la musculatura perivaginal

\begin{tabular}{llll} 
& \multicolumn{1}{c}{ Grado 1} & Grado 2 & Grado 3 \\
\hline & Ligera & Moderada & Firme \\
Presión & 0 & $<3$ & $4+$ \\
$\begin{array}{l}\text { Duración (seg) } \\
\text { Textura tejido }\end{array}$ & $\begin{array}{l}\text { Textura del músculo blanda, } \\
\text { no existe tensión }\end{array}$ & $\begin{array}{c}\text { Textura muscular diferente de la de otros tejidos, } \\
\text { no muy tensa }\end{array}$ & $\begin{array}{l}\text { Tejido muscular con } \\
\text { tensión }\end{array}$ \\
& & & \\
\end{tabular}

Worth et al, 1986

Tabla 4. Test digital para la valoración de la fuerza de los músculos del suelo pélvico

\begin{tabular}{|c|c|c|c|c|}
\hline & Grado 1 & Grado 2 & Grado 3 & Grado 4 \\
\hline Presión & $\begin{array}{l}\text { No } \\
\text { responde }\end{array}$ & Débil & Moderado & Fuerte \\
\hline Duración (seg) & 0 & $<1$ & $>1<3$ & $>3$ \\
\hline $\begin{array}{l}\text { Desplazamiento en el } \\
\text { plano vertical }\end{array}$ & Ninguno & $\begin{array}{l}\text { Las yemas de los dedos } \\
\text { se mueven en sentido } \\
\text { anterior }\end{array}$ & $\begin{array}{l}\text { Todo el dedo se } \\
\text { mueve anteriormente }\end{array}$ & $\begin{array}{l}\text { Todo el dedo se mueve } \\
\text { anteriormente, y es aprisionado por } \\
\text { la musculatura }\end{array}$ \\
\hline
\end{tabular}

Brink et al, 1989.

Tabla 5. Escala de valoración modificada de Oxford para la musculatura del suelo pélvico

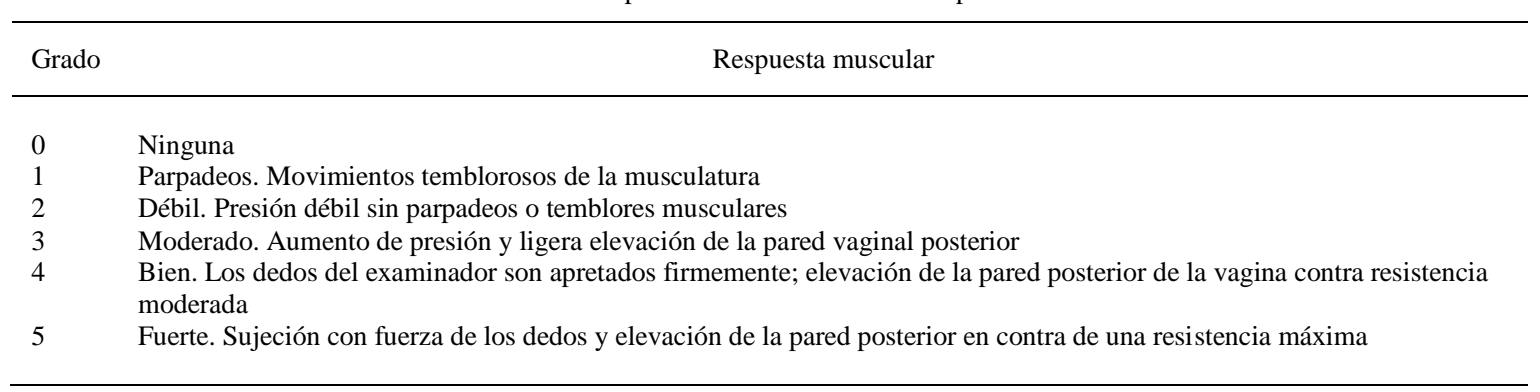

Laycock, 1992.

Pero dentro de la valoración de la contracción muscular, hemos de tener en cuenta otros factores además de la fuerza, tales como la resistencia, la fatigabilidad, y la velocidad de contracción. En este sentido, Laycock ha desarrollado y validado un protocolo de valoración que ha denominado con el acrónimo de PERFECT $(\mathrm{P}=$ power, $\mathrm{E}=$ endurance, $\mathrm{R}=$ repetitions, $\mathrm{F}=$ fast, $\mathrm{ECT}=$ every contraction timed). Este protocolo de valoración tan exhaustivo nos permitirá además establecer el programa de ejercicios de reeducación muscular específico para cada uno de los pacientes, al identificar no solo la 
fuerza de contracción si no también el número de repeticiones y el tiempo de contracción en cada caso. Para valorar la fuerza emplearemos la Escala modificada de Oxford o Escala de Laycock, tanto en el lado derecho como en el izquierdo. Posteriormente seleccionaremos el lado más fuerte para continuar con la valoración del resto de los parámetros de contracción muscular. La resistencia es el tiempo máximo que la paciente es capaz de contraer la musculatura del suelo pélvico con la máxima fuerza de contracción hasta que el músculo se fatigue. Para ello solicitamos a la paciente que mantenga la fuerza cuantificada según la Escala de Laycock hasta detectar una pérdida de fuerza del $50 \%$, con un máximo de 10 segundos. Para valorar la fatigabilidad de la musculatura del suelo pélvico, registramos el número de contracciones con un máximo de 10 que la paciente es capaz de realizar con la máxima resistencia, con tiempo de descanso entre contracción y contracción de 4 segundos. En el caso del parámetro de velocidad de contracción, solicitaremos a la paciente que realice tras un período de descanso de 1 minuto el mayor número de contracciones rápidas de un segundo hasta que la musculatura se fatigue, con un máximo de 10. Las siglas ECT (every contraction timed) completan el acrónimo, y recuerdan que siguiendo el protocolo PERFECT cada una de las contracciones musculares son temporalizadas y registradas, de manera que el programa de ejercicios de cada paciente se realiza de forma individualizada y específica. Así en el caso de que una paciente presente un PERF 4548 será capaz de mantener una contracción del suelo pélvico de grado 4 durante 5 segundos y repetido durante 4 veces, seguido de 8 contracciones rápidas tras un descanso de un minuto. Por el contrario, si la paciente presenta un PERF 2324 nos indicará que es capaz de realizar una contracción con una fuerza máxima de grado 2 durante 3 segundos, y repetido durante 2 veces, seguido de 4 contracciones rápidas tras un descanso de un minuto.

Durante la valoración de la contracción muscular del suelo pélvico, debemos de prestar especialmente atención a la presencia o no de contracciones parásitas, principalmente de los músculos abdominales, aductores y glúteos. Estas contracciones parásitas son especialmente evidentes en pacientes con fuerza de grado 1, o 2, siendo menos frecuentes su aparición en pacientes con grados 4 y 5 según la Escala de Laycock. Para detectar estas sinergias durante la valoración muscular debemos de situar la mano contralateral a la evaluadora sobre el ab- domen de la paciente, con el objeto de palpar cualquier contracción muscular parásita de esta región, a la vez que observamos posibles contracciones a nivel de los muslos y nalgas (fig. 4). Estas co-contracciones pueden cuantificarse y registrarse en la ficha de valoración con una, dos o tres cruces según su intensidad.

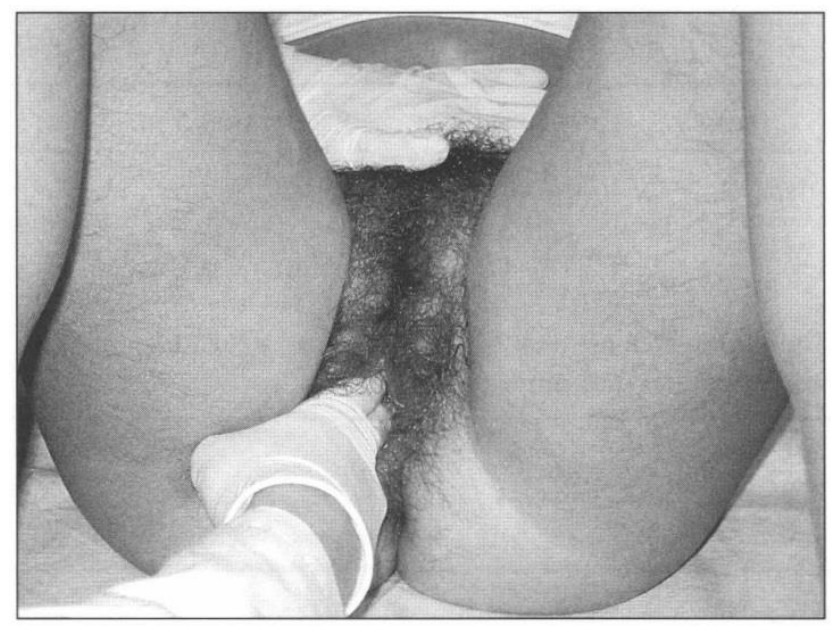

Fig. 4. Palpación de contracciones parásitas abdominales simultáneas a h. contracción del suelo pélvico.

En ocasiones las pacientes al solicitarles una contracción de la musculatura del suelo pélvico, realizan en apnea un empuje caudal sobre el periné. Esto es lo que se denomina Inversión de la orden perineal, y se percibe claramente si al introducir una sonda vaginal y solicitar la contracción del suelo pélvico, ésta es expulsada al exterior. Si bien la aparición de contracciones parásitas es relativamente frecuente, la inversión de la orden perineal solo aparece en el 12-13\% de los casos según Bourcier ${ }^{13}$.

Dentro del protocolo de valoración del suelo pélvico, Dominique Grosse propone la realización del Test Anterior como complemento del Test de fuerza muscular, con el objetivo de evaluar la eficacia 
clínica de la contracción del suelo pélvico sobre la continencia urinaria. En ocasiones podemos encontramos un músculo Elevador del ano con una fuerza de grado 4, y en cambio es totalmente ineficaz en su función de continencia al no conseguir elevar la unión cervicouretral y el cuello vesical. La explicación puede radicar en una lesión neurológica, en una lesión anatómica tras el parto con desgarros del tejido conectivo y fascial, o incluso en una fijación del cóccix frecuentemente en nutación tras el parto.

Para la realización del Test anterior, la vejiga debe estar llena para facilitar la palpación del surco cervicouretral. El fisioterapeura sitúa la yema del dedo índice a nivel de la unión cervicouretral y solicitamos a la paciente una contracción del Elevador del Ano (fig. 5). En el caso de percibir de forma clara la formación de un surco, le atribuimos el grado 2, lo cual significa el ascenso del cuello vesical hacia la sínfisis; grado 1 si palpamos un ligero hundimiento, y grado 0 si no percibimos ni surco ni desplazamiento, en cuyo caso el músculo Elevador del ano es ineficaz sobre la unión vesicouretral.

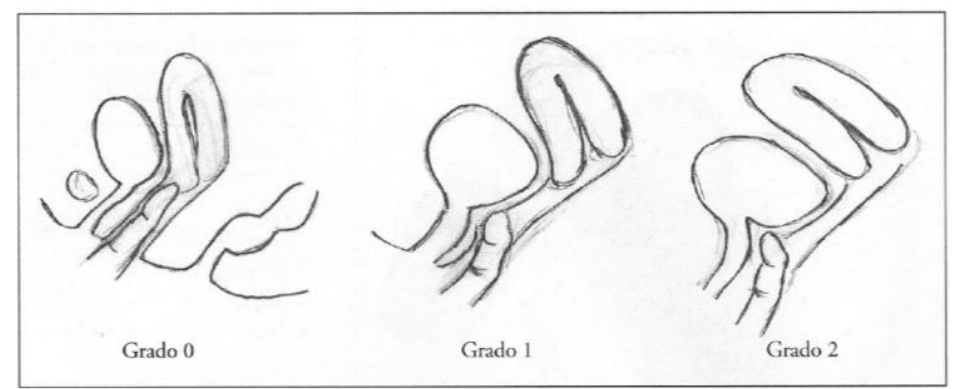

Fig. 5. Grados 0, 1 y 2 del test anterior. Según D. Grosse.

Otro test muy sencillo para evaluar la eficacia de la musculatura elevadora, es mediante una sonda o un tubo de pírex introducido en la vagina. Si al solicitar a la paciente la contracción del suelo pélvico observamos una horizontalidad del tubo, nos indica que el músculo elevador del ano es eficaz en su función elevadora sobre los órganos pélvicos (fig. 6).

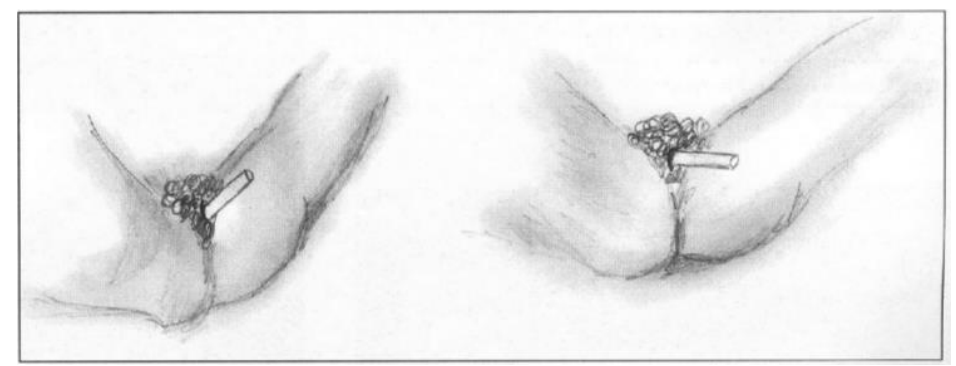

Fig. 6. Horizontalización del tubo de pirex durante una contracción efectiva del suelo pélvico.

Para la valoración instrumental de la contracción muscular podemos emplear un dispositivo denominado perineómetro. Existen perineómetros mano métricos o bien electromiográficos en función de la señal registra- da por la sonda vaginal o anal sea la presión o la actividad electromiográfica del suelo pélvico (figs. 7 y 8). Al igual que en la valoración manual, también podemos aplicar el protocolo de PERFECT en la valoración instrumental, con la ventaja que lo podemos realizar no solo en la posición ginecológica, sino también en sedestación y bipedestación (tabla 6). 


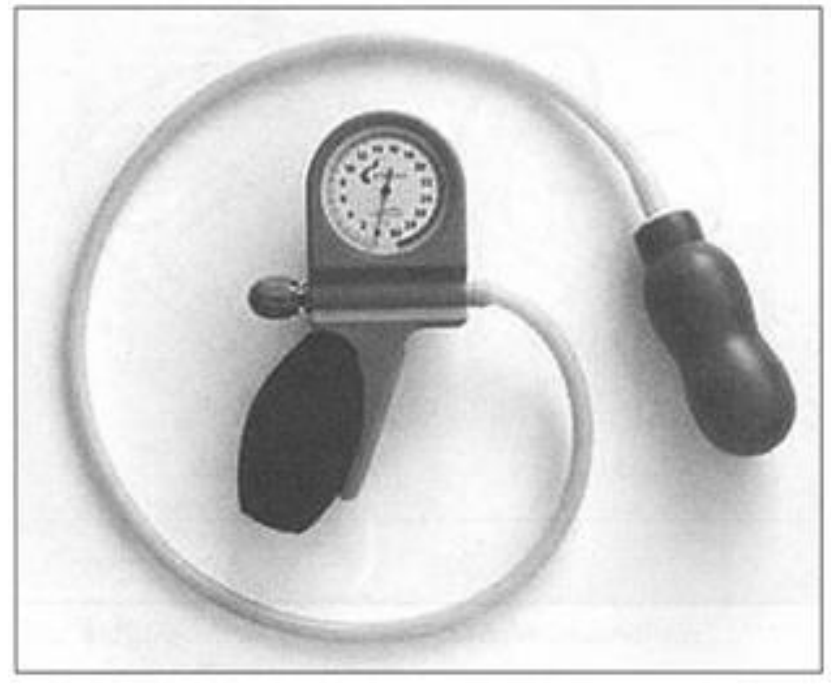

Fig. 7. Perineómetro manométrico.

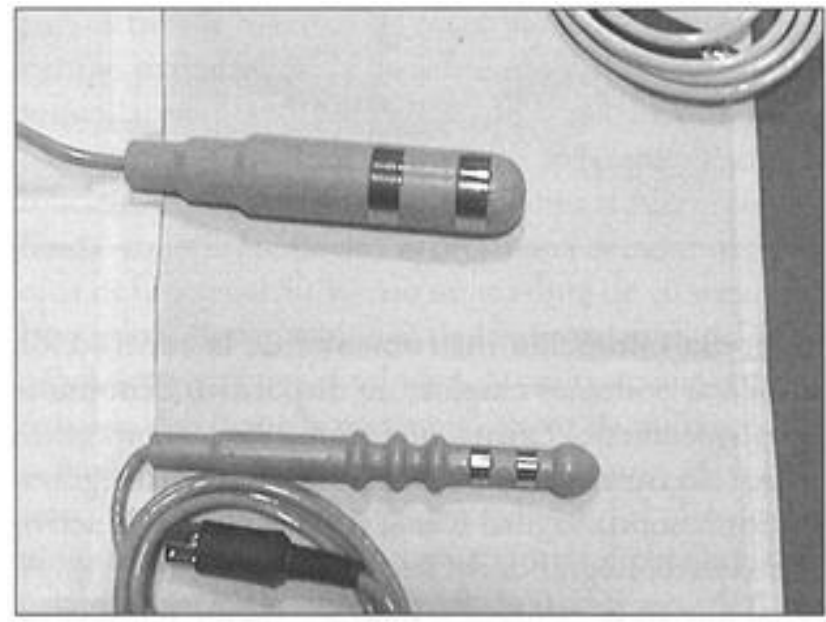

Fig. 8. Electrodo vaginal y anal con captado res de actividad electromiográfica.

Tabla 6. Tabla de equivalencias aproximadas entre la escala de valoración de Laycock y la presión medida por el perineometro mano métrico en $\mathrm{mm} \mathrm{Hg}$

\begin{tabular}{ll}
\hline Perineometro & Escala de valoracion Laycock \\
\hline $0-30$ & Grado 1 y 2 \\
$30-40 \mathrm{~mm} \mathrm{Hg}$ & Grado $3 \mathrm{y} 4$ \\
$50-60 \mathrm{~mm} \mathrm{Hg}$ & Grado 5 \\
$>60 \mathrm{~mm} \mathrm{Hg}$ & Grado $5+$ \\
\hline
\end{tabular}


Dentro de la valoración del suelo pélvico, es preciso identificar la existencia de algún tipo de prolapso de los órganos pélvicos y cuantificar su estadío de evolución. El término de prolapso se corresponde con el descenso parcial o total de alguno de los siguientes órganos a través de la vagina; así, en el caso de que el prolapso se produzca a nivel de la uretra, se denomina uretrocele; si tiene lugar a nivel de la vejiga, cistocele (fig. 9); si el órgano prolapsado es el recto, rectocele, mientras que si son las asas intestinales a la altura del saco de Douglas, elytrocele; finalmente, denominamos histerocele cuando es el útero el que desciende arrastrando en su recorrido las paredes de la vagina. En cuanto a la clasificación del prolapso, éste se clasifica en 4 grados según la situación del órgano prolapsado en relación al introito vaginal; en el caso que se sitúen entre la posición normal y la altura de las espinas isquiáticas se clasifica como Grado 1; Grado II en el caso que se sitúe entre las espinas isquiáticas y el himen; si desciende por fuera del nivel del himen se considera Grado III; y en el Grado IV el prolapso es total.

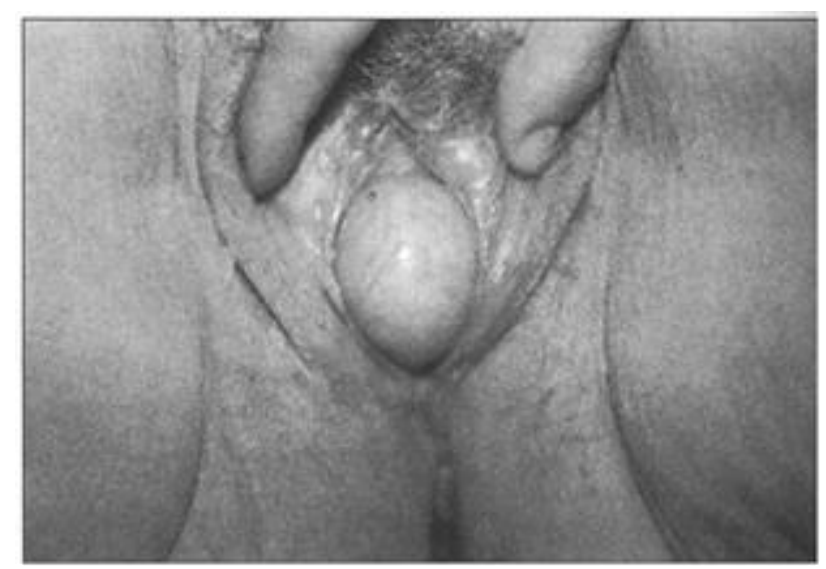

Fig. 9. Cistocele o prolapso de la vejiga urinaria.

Para valorar la presencia de prolapso aplicaremos con nuestros dedos, o bien con las valvas de un espéculo, una depresión en la pared posterior de la vagina; en esta posición solicitaremos a la paciente que realice la maniobra de Valsalva, el gesto de la tos, para poder evidenciar la existencia de un órgano prolapsado, y una maniobra de Aspiración Diafragmática que nos confirme la capacidad de reducción de dicho prolapso.

Durante la Maniobra de Valsalva se aumenta la presión intraabdominal, lo cual provoca un abombamiento de la pared vaginal anterior en el caso de colpocele anterior (fig. 10), o bien de la pared posterior si se trata de un colpocele posterior. Siguiendo el mismo mecanismo, el gesto de la tos provoca el mismo resultado a nivel de los órganos prolapsados. 


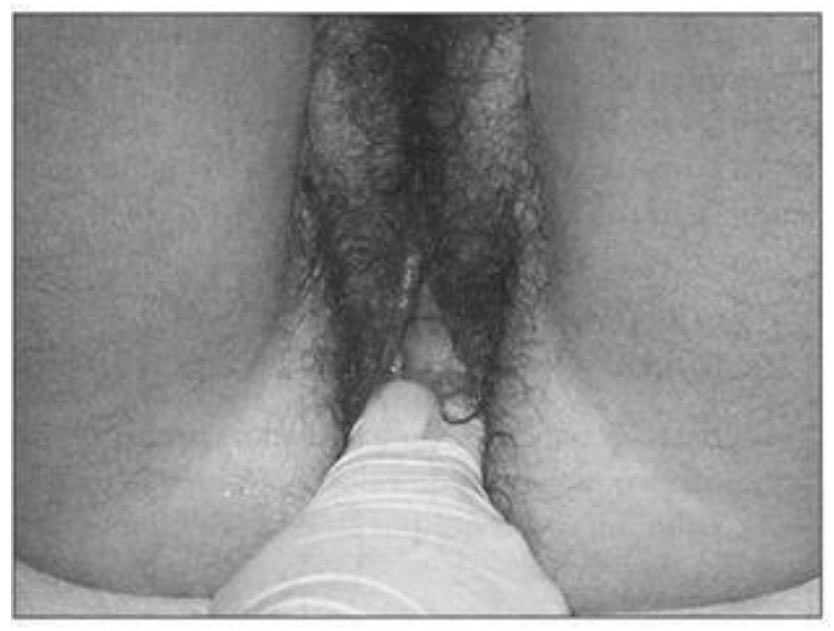

Fig. 10. Depresión de la pared vaginal posterior y visualización de prolapso de la pared vaginal anterior durante una maniobra de Valsalva.

La maniobra de aspiración diafragmática consiste en realizar, tras una espiración lenta y prolongada, un intento de inspirar manteniendo las vías aéreas cerradas. Esta denominada "falsa inspiración" genera una disminución de la presión intraabdominal, una contracción refleja del suelo pélvico, y una succión del órgano pélvico prolapsado en dirección craneal y ventral.

Para una mayor funcionalidad, Laycock propone registrar todos los datos recogidos sobre la palpación, la valoración muscular y la valoración de la estática de los órganos pélvicos, en un gráfico circular denominado "Anillo de la continencia" que representa la vagina". Siguiendo el símil de la esfera de un reloj, el punto de las 12 horas se corresponde con la pared anterior vaginal, las 6 en punto la pared posterior, las 3 en punto la pared lateral izquierda, y las 9 en punto la pared lateral derecha (fig. 11). Dentro de este "Anillo", los prolapsos pélvicos de la vejiga y recto se recogen en forma de semicírculos, de mayor o menor diámetro según el grado de descenso; el uretrocele en forma de "U"; el histerocele se representan por círculos en el centro del anillo de mayor o menos diámetro según el grado de prolapso; las cicatrices en forma de línea en el lateral correspondiente; los puntos de dolor con una $\mathrm{P}$ de mayor o menor tamaño en función de la intensidad del dolor; en cada uno de los lados de anillo, se recoge el grado de fuerza muscular observado en cada lateral.

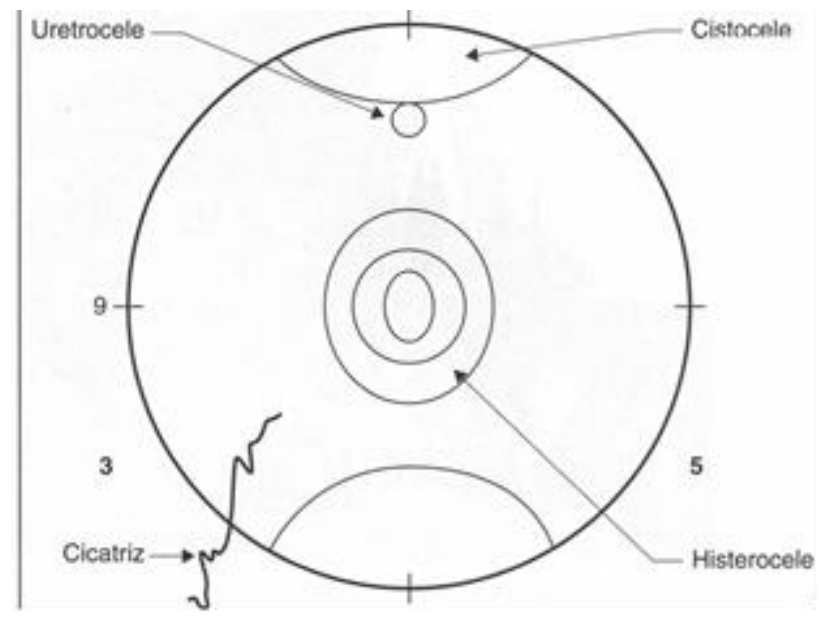

Fig. 11. Anillo de la Continencia. Permite durante la valoración anotar fácilmente todos los hallazgos. En el ejemplo: cistocele, uretrocele, rectocele, histerocele (cada circulo representa un grado), cicatriz en lado derecho, fuerza muscular lado derecho grado 3 , lado izquierdo grado 5. 
Una vez valorada la estática de los órganos pélvicos, realizaremos una serie de Test específicos cuyo objetivo final es evidenciar el tipo y grado de Incontinencia Urinaria. Para ello solicitaremos a la paciente que, en la posición de decúbito supino, realice la maniobra de toser con fuerza y de forma repetida. Hemos de valorar si existe sincronismo entre el esfuerzo y la pérdida de orina, y si dicha pérdida es en forma de chorro, en cuyo caso se trata de una Incontinencia urinaria de esfuerzo. Por el contrario, si aparece un desfase de tiempo entre el esfuerzo y la fuga urinaria con una pérdida abundante de orina, sospecharemos de una Incontinencia urinaria de urgencia. En el caso de que no se produzcan pérdidas de orina en decúbito supino, debemos de realizar este mismo test en posición bípeda, ya que algunos casos de incontinencias solo se manifiestan en posición ortostática.

\section{Valoración neurológica}

Dentro de la valoración neurológica, evaluaremos las afecciones periféricas parciales del suelo pélvico a nivel de los reflejos y de la sensibilidad cutánea, ya que en el caso positivo será apropiada la aplicación de electroestimulación. Para valorar la sensibilidad cutánea podemos deslizar un bastoncillo de algodón por los dermatomas de $\mathrm{S}_{3}$ y $\mathrm{S}_{4}$ en busca de posibles regiones de hipoestesia o hiperestesia (fig. 12). Para valorar la actividad refleja sacra nos basaremos en el Reflejo anal, el Clitoridiano y el Reflejo perineal a la tos. Para valorar el Reflejo anal $\left(\mathrm{S}_{3}-\mathrm{S}_{4}\right)$ podemos emplear un aestesiómetro o un simple "clip", realizando unos leves pinchazos o raspando en el mar- gen anal; en condiciones normales, la musculatura esfinteriana del ano responde con una contracción refleja.

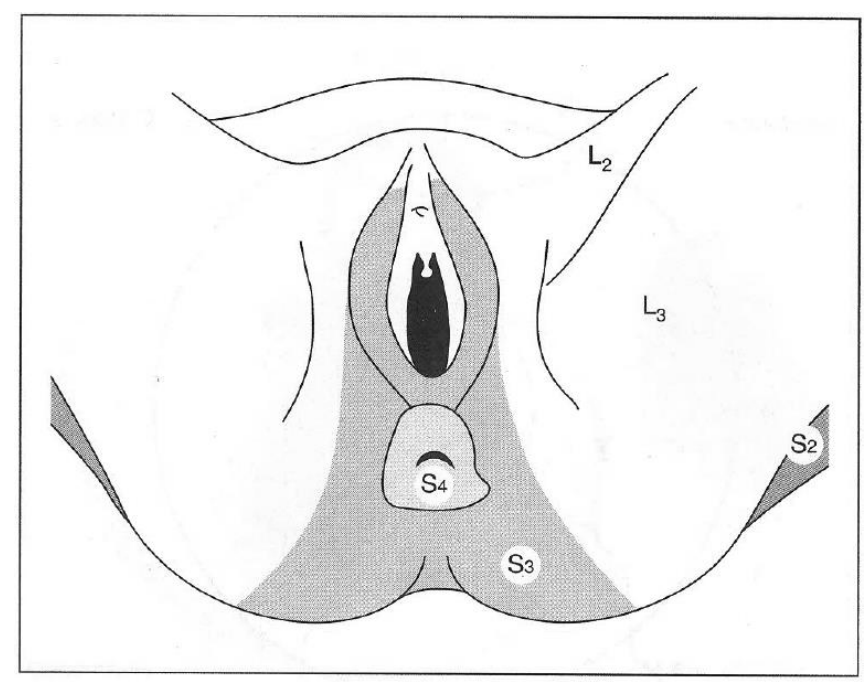

Fig. 12. Dermatomas de S3 y S4

En el caso del Reflejo Clitoridiano (S3) la estimulación del clítoris con un simple bastoncillo de algodón, provoca en el $70 \%$ de los casos pequeñas contracciones visibles de los músculos Bulbocavernosos e isquiocavernosos. Por otro lado, si el Reflejo perineal a la tos $\left(\mathrm{S}_{3}\right)$ está intacto, observaremos una contracción del esfínter anal simultánea al gesto de la tos.

\section{Valoración de la estática lumbo-abdomino-pélvica}

Además de la anamnesis, y la exploración física del suelo pélvico, debemos de valorar también la estática lumbo-abdomino-pélvica por su relación biomecánica con el suelo pélvico.

Desde el punto de vista funcional, el suelo pélvico forma parte de la denominada "Cavidad Abdominal Manométrica", que se define como el espacio delimitado por paredes osteoarticulares y músculoaponeuróticas, con densidades y resistencias diferentes y en el que por definición la presión existente en el interior de la cavidad se considera nula en reposo. Los límites de la cavidad abdominal manométrica son el diafragma torácico en su parte superior, el diafragma pélvico en su parte inferior, la pared abdominal en la parte anterior y laterales, y la columna lumbar en la pared posterior. 
Durante un esfuerzo, los vectores de presión resultantes no son idénticos en cualquier punto de la cavidad abdominal manométrica debido a la diferente densidad y resistencia de sus paredes. Así, en el caso de una cincha abdominal competente y una lordosis lumbar fisiológica, un aumento de la presión intraabdominal provoca vectores resultantes en dirección a la región abdominal y a la región posterior del periné y al sacro. Sin embargo, si la cincha abdominal está hipotónica o bien existe una hiperlordosis lumbar, se produce un desplazamiento anterior de la línea ombílico-pubiana, con el resultado de una hipertransmisión de vectores resultantes hacia la región del periné anterior, que progresivamente irá perdiendo la capacidad de sostén de los órganos pélvicos (fig. 13). Este mecanismo favorece una hipotonía de la musculatura del suelo pélvico, la aparición de prolapsos pélvicos, y un problema de incontinencia urinaria potencial.
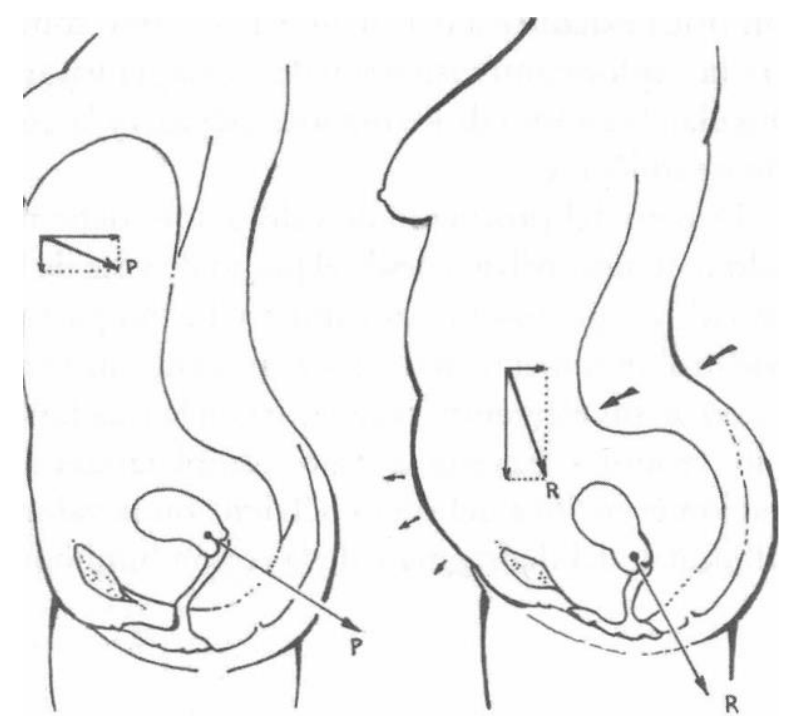

Fig. 13. Trammisión de los vectores de foerza resultantes durante un aumento de 14 presión intraabdominal. P: 1kctor resultante en caso de pared abdominal tónica, se dirige a zona posterior. R:

Véctor resultante en pared abdominal hipo tónica e hiperlordosis lumbar, se dirige a periné anterior.

Por lo tanto, deberemos de valorar los siguientes aspectos biomecánicos de la cavidad abdominopélvica: el tono de la cincha abdominal (oblícuos y transverso del abdomen), la presencia de diástasis abdominal, es decir, la separación de los vientres musculares del recto anterior del abdomen, el grado de hiperlordosis lumbar y de acortamiento del músculo Psoas-Ilíaco, así como el bloqueo del diafragma en posición inspiratoria.

Para valorar el tono de la cincha abdominal, solicitaremos a la paciente una inspiración abdominal profunda y palparemos el grado de resistencia que ofrece este músculo al estiramiento.

Por otro lado, la dimensión de la diástasis abdominal es siempre un reflejo del grado de hipotonía de la cincha abdominal. Para valorar el grado de diástasis, situaremos las yemas del dedo índice y medio a nivel umbilical e in- vitamos a la paciente a realizar una flexión anterior de la cabeza y cuello (fig. 14). El test será positivo cuando la separación entre los dos vientres musculares de los rectos anteriores supere $\operatorname{los} 2,5 \mathrm{~cm}$. Este test se realizará a continuación a nivel supra e infraumbilical. 


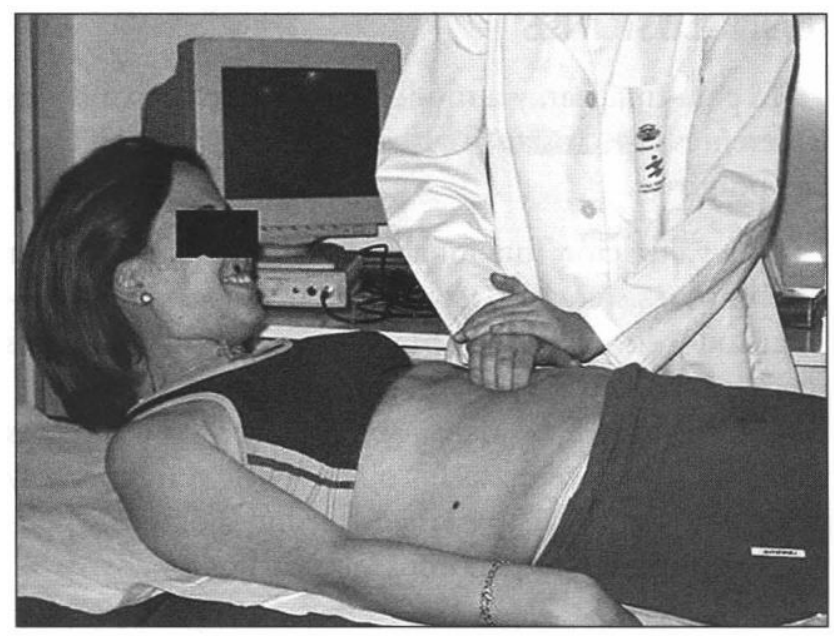

Fig. 14. Valoración de 1a diástasis de los rectos anteriores del abdomen.

En el caso de que el diafragma se encuentre hipertónico, estará descendido en una posición similar a la de inspiración. En esta posición cualquier esfuerzo resultará hiperpresivo a nivel de la cavidad manométrica. Si el diafragma está hipertónico palparemos una resistencia al intentar introducir los dedos por debajo de la arcada costal (fig. 15). Si el diafragma está hipertónico habrá que relajado para disminuir la hiperpresión.

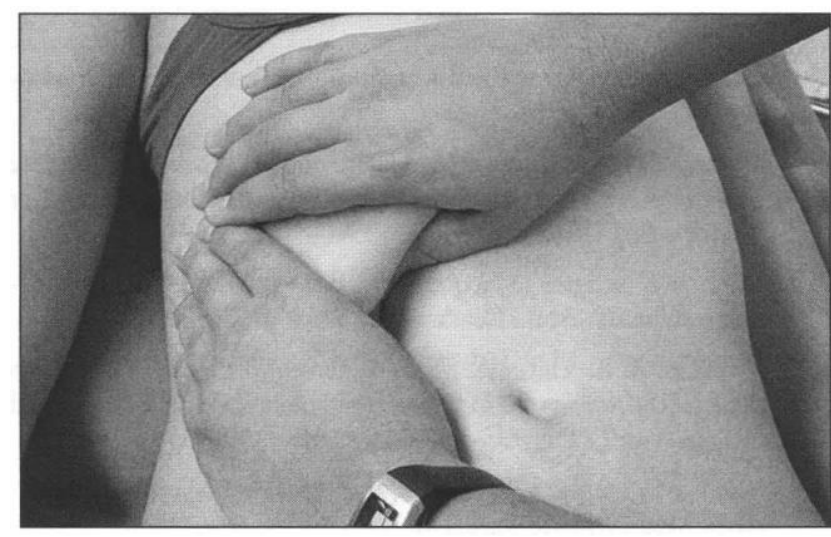

Fig. 15. Valoración del tono del diafragma

Los datos obtenidos en el conjunto de procedimientos cualitativos y cuantitativos que comprenden la entrevista clínica y la valoración funcional del suelo pélvico se recogerán de manera ordenada en hojas de registro. 


\section{Conclusiones}

Ya para finalizar, y a modo de reflexión acerca de los diferentes aspectos analizados, señalaremos los siguientes puntos:

- La valoración funcional del suelo pélvico ha de ser un proceso sistemático y ordenado que nos permita evaluar rodos los elementos biomecánicos fundamentales para la intervención fisioterápica.

- A partir de estos datos obtenidos en las diferentes pruebas, estableceremos los objetivos de tratamiento y seleccionaremos las técnicas de reeducación pelvi-perineal más adecuadas para conseguir dichos objetivos.

- Además de la anamnesis, realizaremos una exploración física exhaustiva del suelo pélvico, que comprenderá la exploración visual y palpatoria, la valoración muscular, la estática de los órganos pélvicos y la exploración neurológica.

- Dentro del protocolo de valoración, debemos de analizar el suelo pélvico desde el punto de vista de la globalidad, ya que biomecánicamente forma parte de la cavidad abdominal mano métrica en donde disfunciones en uno de sus elementos repercutirá en la funcionalidad de los restantes. En este sentido, completaremos la valoración específica del suelo pélvico con la valoración abdominal, del diafragma y de la región lumbopélvica.

\section{Bibliografía}

1. Grosse D, Sengler J. Reeducación del periné. Barcelona: Masson, 2001.

2. Wilson PD, Herbison RM, Herbison GP. Obstetric practice and the prevalence of urinary incontinence three months after delivery. Br J Obstet GynaecoI1996;130(2):154-61.

3. Minaire P, Sengler J, Jacquetin B. Épidémiologie de l'incontinence urinaire. Anna Réadap Med Phys 1995;38: 1-8.

4. Samuelsson E, Victor A. Signs of genital prolapse in sweadish population women 20 to 59 years old of age and possible related factors. Ann J Obstet Gynecol 1999; 180:299-305.

5. Minaire P, Sengler J. Epidémiologie de l'incontinence. Ann Réadap. Med Phys 1995;38:1-8.

6. Rorveit G, Kjersti A, Hannestad Y, Hunskaar S. Urinary incontinence after vaginal delivery or caesarean section. The New England Journal of Medicine 2003;348(10):900-7.

7. Minaire P, Lyonnet A, Sabot E. Rééducation périneale er srarique lombo-pelvienne. Ann Kinesither 1988;15:3914.

8. Valancogne G, Caufriez M, De Gasquet B, Guillarme L, Dumonr P. Rééducation perineologique et pressions dans l'enceinte manométrique abdornino-perinéale (comunicación). En: abstracts Congres de la SIFUD. Lyon, 2001.

9. Elleuch M, Ghattassi J, Guermazi M. Stress incontinence in nulliparous sportswomen: an epidemiological inquiry coneerning 105 cases. Annales de Readaption et de Medicine physique 1998;41 (8):479-84.

10. Rud T. The effects of oestrogens and gastagens on the urethral pressure profile in urinary continent and stress incontinente women. Acta Obster Gynecol Scand 1980;59:265-70.

11. Surhersr J, Brown M. Assessing the severity of urinary incontinence in women by weighing perineal pads. The Lancet 1981;23:1128-30.

12. Jeyaseelan S, Haslam J, Winstanley J, Roe B, Oldham J. Digital vaginal assessment, an inter-tester reliability study. Physiotherapy 2001;87(5):243-50.

13. Bourcier A. Le plancher pelvien. Paris: Éditions Vigor, 1991.

14. Laycock J, Haslam J. Therapeutic Management of incontinence 